\title{
Pharmacoeconomic analysis of ozone therapy supported by agent based process simulation and data mining
}

\author{
Marco Remondino, ${ }^{1-3}$ Luigi Valdenassi, ${ }^{2,3}$ Marianno Franzini ${ }^{2,3}$ \\ ${ }^{1}$ Department of Economics, University of Genoa; ${ }^{2}$ Department of Internal Medicine and Medical Therapy, University \\ of Pavia; ${ }^{3}$ Italian Scientific Society of Oxygen-Ozone Therapy (SIOOT), Gorle (BG), Italy
}

\begin{abstract}
Pharmacoeconomics is the set of methods applied to the economic evaluation of pharmacological treatments. Its goal is to determine the best possible health outcome with the available resources by comparing costs (inputs) and the consequences/benefits (outcomes) of one or more alternative choices. Ozone therapy can be considered as a powerful alternative to traditional methods in the management and treatment of several diseases, like, e.g. rheumatoid arthritis, lumbago, disc hernia, knee and hip disease, fibromyalgia, stroke outcomes, neurodegenerative syndromes and more. It is thus a very interesting topic to compare the costs and outcomes of ozone based treatments with those coming from other alternative methods, with the perspective of pharmacoeconomics. Several methods have been proposed for pharmacoeconomical analysis, including statistical and simulation tools. The purpose of this research paper is to review some of the simulation tools that could be used in this field and in particular to propose a hybrid simulation method, defined as agent based process simulation, which considers not only the involved costs, but also other non-monetary
\end{abstract}

Correspondence: Marco Remondino, Scientific Society of OxygenOzone Therapy (SIOOT), via Don Luigi Sturzo 2, 24020 Gorle (BG), Italy. Tel:. 035.19910105.

E-mail: info@ossigenoozono.it

Key words: pharmacoeconomics; data mining; agent based process simulation.

Contributions: all authors contributed to this article, both substantively and formally. In particular, the paragraph entitled Ozone therapy: a brief review of main positive effects for the medical field and its subsections are to be attributed to LV and MF, while all the rest of the work is to be attributed to MR.

Conflict of interest: the authors declare no potential conflict of interest.

Received for publication: 23 April 2018.

Accepted for publication: 24 April 2018.

(C) Copyright M. Remondino et al., 2018

Licensee PAGEPress, Italy

Ozone Therapy 2018; 3:7518

doi:10.4081/ozone. 2018.7518

This article is distributed under the terms of the Creative Commons Attribution Noncommercial License (by-nc 4.0) which permits any noncommercial use, distribution, and reproduction in any medium, provided the original author(s) and source are credited. outcomes. In the same vein, a data mining set of techniques is proposed to collect data and to feed the simulation model.

\section{Introduction}

Pharmacoeconomics ${ }^{1}$ is the set of methods applied to the economic evaluation of pharmacological treatments. It may be defined as a tool that allows choices to be made using explicit and rational criteria. According to the traditional approach, the evaluation of a pharmacological therapy is made in the light of three clinical indicators: tolerability (safety), clinical efficacy (efficacy), and epidemiological efficacy. The first checks for the presence or absence of side effects related to the administration of treatment, the second determines whether the condition of patients' treatment improve in function of the therapy administered, and the third checks whether the treatment can be used advantageously in general medical practice. The economic approach, i.e. pharmacoeconomic evaluation, adds to the three indicators of the traditional approach the principle of allocative efficiency, thus shifting the analysis also on the cost side with the aim of determining the best possible health outcome with resources (scarce) available. It should be remembered that allocative efficiency means the convenience in allocating scarce resources between possible alternative uses (therapeutic treatments).

The goal of pharmacoeconomy ${ }^{1}$ is therefore to determine the best possible health outcome with the available resources by comparing costs (inputs) and the consequences/benefits (outcomes) of one or more alternative choices. Components of the economic evaluation are therefore costs (input) and consequences or benefits (outcomes). Costs are generally divided into direct, indirect and intangible and are always expressed in monetary units. The benefits may also be directly expressed in monetary units, or may be indicators of intermediate or final result and therefore expressed, e.g. as arterial pressure, lives saved, cases diagnosed, cases avoided, Life Years, Quality Adjusted Life Years, and so on.

Ozone therapy is one of the modern non-drug treatment methods. It has been used for more than 100 years. Medical reports on the successful use of ozone in the treatment of various diseases and studies on its effects have led to a rapidly growing interest in it. Some other factors were responsible for the wide spread, such as the simplicity of performance, the good tolerability by the patients, the absence of side effects or side effects and the high medical-social and economic efficiency. In this specific field, it is of great interest to compare the outcomes and costs of a traditional therapy with those of a therapy based on ozone.

In such a complex scenario, where it would be useful to evaluate not only the economical difference among treatments, but also social and health factors have to be taken into account, a very 
interesting tool to use would be simulation. While there exist many simulation paradigms, two of them seem optimal for this task: discrete event process simulation (for its capability of reproducing a well-known situation with process based blocks) and agent based simulation (for the possibility of building models with a bottom-up approach, by defining the interaction rules and letting the aggregate behaviour to emerge in a spontaneous way. The combination of the two is proposed in this paper, with the aid of a data mining technique, in two different perspectives: ex ante, to help collecting data to be used in the simulation model; ex post, to help analysing and selecting data coming from the simulation.

\section{Pharmacoeconomics: the scenario}

The economic assessment of a treatment is undertaken for two fundamental reasons. The first is that the objectives of public institutions, including the health of citizens, may not be measurable directly by optimising the profit of some producers, but require the composition of interests, sometimes conflicting, in order to achieve the highest possible collective welfare. The second is the presence of numerous distortions and imperfections that affect the prices of both inputs and goods in the health market, both private and public (so-called market failures).

This is because the pharmaceutical sector is moving away from a competitive market, so much so that it can be defined as a nonmarket, in fact the presence of an uninformed consumer, i.e. who is not able to make choices that express her preferences and who, in any case, does not have of the necessary information on the efficacy/safety of the pharmacological alternatives on the market, the function of the doctor who decides for the patient and social demand shifting the burden from the consumer to a paying third party allows the producer to raise the price above the break-even point and the consumer to demand more expensive quantities of goods and services than would be the case in a context of perfect competition. So, since resources are scarce by definition, it is not sufficient for a drug to be effective to justify its administration, the costs should also be estimated economics that it induces in order to be able to choose between different alternative uses of the resources themselves. Pharmacoeconomics ${ }^{1}$ is therefore the set of methods applied the economic evaluation of pharmacological treatments, can therefore rightly be defined as interaction between clinical and economic evaluation.

As to the involved costs, pharmacoeconomic classifies costs differently according to their nature. The costs can be divided into three main categories: direct costs (health and non-health), indirect costs (health and non-health), and intangible costs. Direct costs are associated with the resources used by the programme. They are divided into direct health costs and direct non-health costs. Direct health costs include all costs incurred by the National Health Service and third party payers (e.g. private insurance); they are represented by resources used in prevention, diagnosis, and therapy and rehabilitation (e.g. drugs, medical materials, examinations laboratory equipment, hospitalisation, etc.). Direct non-health costs are those costs related to the resources of non-health nature consumed as a result of the programme. Usually these are the ones supported by the patient (such as transport costs and the costs of access to social assistance services). Indirect non-health costs represent the value of resources that are not produced by the disease, either by the patient or by other (family members), as the latter do not provide professional care; they therefore represent the reduced or lost productivity of patients and their families. The classic example is days off work and/or loss or loss of working ability. Indirect health costs are the costs that will have to be borne in the future due to the increased survival (life expectancy) of the patient as a result of the treatment programme. Finally, intangible costs describe the costs associated with the physical and psychological distress associated with the morbid condition. Often these costs, due to obvious problems of valorisation, are not quantified in monetary terms, but converted into measurements of the reduced quality of life of patients.

An important step in the evaluation of costs in pharmacoeconomics is the process of attributing the appropriate unit cost for each resource considered in the analysis. For all those resources (e.g. drugs and medicines) whose cost corresponds to a purchase price on the market, the operation of valorisation is rather simple, while for the other resources, which do not have a specific reference in the market, different considerations apply. An alternative method, which helps in the absence of market prices, is to use the Fares (if available). For example, the Diagnosis Related Group reimbursement rates are available for hospitalisation, while for the vast majority of other medical/ambulatory services there is a rate (National or Regional). However, it should be noted that the use of tariffs creates problems; in fact, tariffs rarely coincide with the real cost of the services to which they refer, since they can usually be values determined by an agreement between the supplier and the lender, or estimates of values on a sample basis, or adjustments to historical values previously agreed. The so-called human capital method is a reference method for estimating indirect costs, which are considered as total loss of production. On the basis of this approach, in the case of impossibility to work due to sickness, the loss of production may be calculated using an estimate of the average daily gross income of the worker absent due to sickness.

\section{Ozone therapy: a brief review of main positive effects for the medical field}

Medical reports on the successful use of ozone in the treatment of various diseases and studies on its effects have led to a rapidly growing interest in it. Even if the purpose of this paper is not analysing the effectiveness of ozone, it's worth mentioning that ozone has proved to have several positive effects and interactions that could be employed in the medical filed. Here follows a nonexhaustive list. ${ }^{2}$

\section{Effects on metabolism}

Increase in glycolysis delta: the high amount of energy released from the aerobic demolition of glucose is used for the synthesis of ATP, but it is clear that the efficiency of the process depends on the continuous supply of oxygen. There are circumstances in which the demand for ATP by different organs increases. In these cases, the sudden increase in ATP demand can only be partially met by an increase in the speed of mitochondrial oxidative processes and the oxygen supply remains insufficient to meet demand. Under these conditions, glycolysis, which can meet the demand for ATP to ensure the energy requirements necessary to maintain cellular homeostasis and the required performance. This makes it clear that the acceleration of glycolysis since ozone, following its action on coenzymes, leads to increased ATP formation.

Activation of Lipid metabolism: this effect ascribable to ozone is aura- towards an intervention on fat catabolism and for a direct lipolytic effect. All this has the consequence of an increased production of energy that is useful in all these conditions, especially physiological, where an increase in work is required.

\section{Effects on red blood cells and microcirculation}

The erythrocyte membrane is composed structurally of proteins, carbon hydrates and phospholipids. The first phase of the reaction is represented by the reaction of ozone with the double bonds of unsaturated fatty acids of the membrane of erythrocytes 
(electrophilic addition), so short chain peroxides penetrate into the erythrocytes themselves, influencing their metabolism. This means more sugar being broken up and the most important product of this reaction chain is diphosphoglycerate, which, as a deoxygenating substance, is the key element in the therapeutic effect of ozone by moving the haemoglobin dissociation curve to the right. On the other hand, the formation of peroxides would lead to an increase in hydrogen ions in the hell of red blood cells, since the oxygenated hemoglobin behaves like a stronger acid than the deoxygenated hemoglobin, this change in $\mathrm{pH}$ by varying the affinity of $\mathrm{Hb}$ for oxygen would lead to a greater transfer of the same (Bohr effect).

\section{Action on rheology}

The administration of ozone induces, at low concentrations, a reduction in the overall blood viscosity and platelet aggregation. These erythrocyte modifications are testified by an increase in the erythrocyte filterability in the smaller capillaries, where the emazie instead of moving disorderly align and proceed along the axis of the vase by placing themselves in a stack (Fahraeus-Lindquist effect), increase the deformability thus facilitating metabolic exchanges.

\section{Bactericidal fungicidal and virus static action}

The bactericidal and fungicidal action derives from the direct contact between the ozone and the microorganisms and is due to the destruction of the capsule subjected to the oxidative process determined by the gas and/or peroxides. The virus action of ozone and its peroxides is carried out instead by the cellular receptors present on the viral surface in such a way as to prevent contact between vims and the target cell, so that the entire replication cycle is stopped. In fact, the penetration of cell vims is possible because the acceptors of vims are made up of $\mathrm{N}$-acetylglucosamine while the cellular receptors are made up of $\mathrm{N}$-acetylneuraminicol.

\section{Action on the immune system}

It has been observed that oxidising agents such as hydrogen peroxide can stimulate the activation of lymphocytes or monocytes causing the release of cytokines including: interferons, tumour necrosis factor and interleukins. It was concluded that this gas is capable both in vitro and in vivo of determining a blood-verifiable immunomodulating effect with the gradual reduction of serum immunoglobulins and the rapid fall in the clearance of circulating immune complexes. Laboratory data allow to highlight an inductive role by the $\mathrm{O}_{3}$ on the production of different cytokines. All this opens a wide field of research on the possible therapeutic developments of $\mathrm{O}_{2} \mathrm{O}_{3}$ in the treatment of different diseases of immune origin.

\section{Analgesic and anti-inflammatory action}

Therapy with ozone leads to a rapid disappearance of joint pain and oedema and a normalisation of local temperature, with increased mobility and articular function. These effects are probably due to the inhibition of proinflammatory prostaglandin synthesis or the release of bradykinin or halogen compounds; neutralisation of reactive oxygen species by stimulating local production of antioxidant enzymes; inhibition of the release of proteinase from macrophages and neutrophil polymorphonucleates; modulation of the production of proinflammatory cytokines. The effect of ozone on the components of the intravertebral disc is complex and very excused. It is believed that ozone has positive effects on both the protein component and on the glycemic one and on the water contained in the polposo6 nucleus. In particular, an activation of the local metabolism could be realized to increase local oxygenation and a direct effect on the altered components of the hemiatol inter- vertebral disc. This would explain the reason for the positive results after ozone therapy in treating chronic situations very different from each other in terms of pathological constitution and localization6 In addition, different diseases related to the musculoskeletal system can benefit both when it comes to inflammatory and degenerative events. This in relation to a series of linked effects that, starting from an improvement of the local micro vascularisation, could cause the removal of algogenic substances with relative tissue disinflammation, reduction of contracture and oedema, recovery of rachis mobility and therefore the arrest of the pathophysiological mechanisms of degeneration.

\section{Uses in treatments for several diseases}

Considering all this, ozone has been and is used successfully to treat several diseases. Some of those are: hepatitis $\mathrm{C}$ acute and chronic liver disease, chronic liver disease (hepatitis C and B) cirrhosis, hypercholesterolemia, lymphedema, colibacillosis, eczema anal hemorrhoids, proctitis, constipation, ulcerative colitis (Crohn disease), gastritis, gastric and duodenal ulcer, herniated disc, conflicts discorradiculares, arthritis, rheumatic, periarthritis, fibromyalgia, chronic fatigue syndrome, multiple sclerosis, carpal tunnel, backache, back pain, sciatic pain, osteoarthritis, osteomyelitis, vertebral osteochondrosis, gonarthrosis, spondylarthritis, ankylosing spondylitis, synovitis, tendonitis, bursitis, trochanters, hip osteoarthritis, epicondylitis (tennis elbow), pubalgia, ischial bursitis, post-surgical fibrosis, etc., migraine, trigeminal neuralgia, post-herpetic neuralgia, circulatory headache, cluster headache, Parkinson's disease, depression, phlebology and angiology, venous insufficiency, diabetic ulcer, diabetic foot, decubitus sores (bed sores), varicose veins, spider veins, degenerative vascular diseases (arteriosclerosis obliterans), lymphangitis, furunculosis, recent thrombophlebitis, pathologies flebiáticas, gangrene, etc., ischemic heart disease, cardiac stenosis, angina pectoris, venous hypertension syndrome, hypertension, etc., adjuvant radiotherapy and chemotherapy in cancer, helping in the acquired immunodeficiency syndrome (AIDS), senile dementia, Alzheimer's, arteriosclerosis, cerebral sclerosis and Parkinson's, open-angle glaucoma, optic neuropathy, retinitis pigmentosa and AMD, gingivitis, alveolitis, cloceovestibular syndrome, chronic tonsillitis, pharyngitis infectious, peripheral vestibulocochlear syndrome, Chronic pelvic inflammation, recurrent vulvovaginitis, genito-urinary infections by viruses, fungi / bacteria, mastodinea, menopausal syndrome, puerperal infections and inflammations, celulite, Herpes simplex and zoster, acne, eczema, Herpes sores, skin virus, burn / wound healing, alopecia, psoriasis, dermatitis.

\section{Simulation}

According to Ostrom, ${ }^{3}$ simulation is a powerful alternative to the other two symbol systems: verbal and mathematical reasoning. Simulation has an advantage over the other two, which lies in high portability on a computer, through a specific program or tool. Using computer programs, quantitative or qualitative situations can then be modelled and analysed. While there are many different formal approaches to computer simulation, two of them seems particularly interesting for pharmacology, both of which lead to the creation of a computer model of a complex system: ${ }^{4}$ i) Discrete event, process simulation; through which models of known parts of the systems are created and simulated. Its greatest advantage is that it starts with a basic schema that is often derived from simple observations of the real situation, which greatly facilitates the integration of an actual system into a process simulator. This approach 
is widely used and allows to analyse part of a set and to examine its behaviour using a what-if analysis. This is why process simulation is of great help in decision-making. Unfortunately, there is no universal modelling language for process simulation, which often requires deep translations to port models from one tool to another. The second disadvantage is that to simulate a process with this approach, it must be well known in all its parts; if part of the process is uncertain, then it is impossible to validate a simulation as a model of the real world to represent. Moreover, this method is quite static, which means that the relationships between the different parts involved in the model must be well described and that there is no possibility of self-organization; ii) Agent-based simulation; in pharmacoeconomics, it is often very difficult to describe in detail the different parts and the whole..$^{5}$ For this reason, discrete event simulation of processes, at least alone, does not seem the ideal tool to model these complex environments, where not only costs are relevant, but also other outcomes, like non-monetary indicators (e.g. life expectancy, life quality and so forth). On the other hand, there are formalisms that make it possible to investigate the state of emergency of social behaviour by creating and exploring models. Thanks to the ever increasing computing power, it is possible to create software based on intelligent agents whose aggregated behaviour is often complex and difficult to predict and which can be used in open and distributed systems. A software agent can be described as a flexible system capable of dynamic and autonomous actions to achieve its design objectives located in a given environment. The main characteristics of a software agent are: the ability to carry out actions according to a certain external contribution, which in turn can change the environment itself; autonomy in the execution of actions, without human intervention; flexibility and adaptability. Some specific agents can also be proactive, meaning they are targeted and social, as they can interact with other artificial agents, robots and humans.

\section{Data mining}

Data mining ${ }^{6}$ is the key element of the Knowledge Discovery in Databases (KDD) task. KDD is defined as the process of identifying valid, novel, potentially useful and ultimately understandable patterns in data. Finally, we could add that such a task typically involves a large amount of data that is typically stored in analytically oriented data stores called data marts. Data mining is not a field in itself; it is rather a collection of methods of data analysis from various areas of computer science, artificial intelligence and statistics. Statistics in particular provide mathematical concreteness for many of the methods of date mining. Data mining was born in the last decades to help users gain useful knowledge from the otherwise overwhelming amount of data coming from both the web and the data collected daily by companies. The type of knowledge that users can gain from the raw data is heterogeneous and depends above all on the type of data available.

The type of data and the type of task guide the data analysis process itself, i.e. rather the production of an artigianalized process guided by experience than the result of an automatic process. The types of tasks that data mining could perform can be roughly divided into two categories. ${ }^{6,7}$ Forecasting and descriptive tasks. The first type of tasks tries to discover a model that controls the behaviour of some variables in a system to predict such values in zones not covered by the examples. The second type of task tries to find some categorizations of the data that generate a shrunken descriptor for wider data segments.

\section{Predictive data mining}

One of the predictive tasks of data mining is to find a form of classification of the elements contained in the data mart from a set of raw data. If there is a finite set of classes that describe the domain of the data, classification can be performed by some ifthen rules that help the user to classify a new element in such a predefined class.

Such a classification process is based on the values of some characteristics of the item itself and can be deterministic (e.g. there is no doubt that the item belongs to the given class) or heuristic (e.g. the assignment of the item to one or more classes is given with a certain degree of certainty). The association model extracted so far can take the form of a decision tree instead of a set of if-then rules, but the purpose of the model retrieved remains the same. If the classification domain is not finite (for example, if the variable interested in the prediction process is a real number), the operation is called regression. The regression task helps the user to model an analytical function that describes the data set passed to the task and can predict new, un-transmitted values.

\section{Descriptive data mining}

Descriptive data mining is about discovering interesting regularities in the data, uncovering patterns and finding interesting subgroups in the mass of data. This type of data mining leads to a categorization of the original amount of data that was not recognizable before the task was executed. The domain expert must then interpret the patterns revealed so far to explain them. A typical product of this type of task is the discovery of association rules that find immature relationships between the values of characteristics when looking at the examples proposed as training. Such association rules can be used as classifiers to find some subgroups that divide the population into relevant clusters. The clustering reflects an important division in the data that could be decisive in using a small number of stereotypes instead of a large number of single points. Another important task in connection with data mining is the use of advanced visualization techniques. Since data analysts and domain specialists do most of the discovery work, it is very important to find good visual metaphors to give users the right intuition for analysis.

Of course, such metaphors are only useful to guide intuition, to ensure mathematical soundness, data mining is supported by statistical methods such as probability laws for the prediction of item values, Bayesian theorems for the definition of some kind of causality, and so on. The techniques of data mining, which are based on statistics, require a large number of elements in order to achieve satisfactory results. If only a few examples are available, techniques of machine learning from AI and inductive logic are proposed. Such techniques are based on symbolic reasoning and non-classical logic and do not require statistical tools to verify solidity.

\section{Agent based process simulation fostered by data mining}

In the field of pharmacoeconomics, the interactions among basic subsystems are usually very complex, and could involve human or non-deterministic participation. That would be very difficult, or even impossible to represent, with a straight discrete process based simulation model; that is where agent-based connections between the subsystems can be used. ${ }^{4,5}$ These agents could simulate the complex behaviour of systems where decisions take place, with certain rules or through artificial intelligence patterns. The agents ${ }^{5}$ should be quite simple, but structured, capable of acting based on stimuli from the environment (i.e. the output of a subsystem modelled with a process-based approach), and around an output that influences the functioning of other subsystems. Besides, certain phenomena (like diffusion, or emerging complex behaviours) are definitely easier to simulate with an agent-based approach. When the object of study is very complex, not all parts are capable of being strictly deterministic, in the sense that they 
can be influenced by unforeseen influences from the environment. With the help of a process-based approach it is possible to model the deterministic, process based parts. By employing agent-based rules, it would then be possible to create a more realistic model of the complex system to be simulated.

The data for the simulation model built with this approach are to be selected by data mining. ${ }^{6}$ In particular, not all data coming from ozone-based treatments can be useful for the model. Whereas in discrete event process simulation the emphasis is on the functional description of the modelled parts in detail, in agent-based simulation the most important facet is the interaction among those parts. As a matter of fact, it is interaction that produces a variety of behaviours that have could even not explicitly be described in the model of the individual parts. In agent-based simulation, there are therefore two main areas levels that use different data, with different goals. A micro level to describe a simple local behaviour and a macro level, whose effects partly result from the micro level and partly from the interaction of more elements. Such emerging behaviours could be revealed by non-explicit patterns in the simulation data and a subsequent phase may be needed for simulation to reveal the model that subdivides the data. Data mining techniques can therefore be the key to unveiling non-trivial knowledge through the initial assumption used to build the micro level, the model and structure of the agent aggregation that emerged from the simulation. Data mining and machine learning in general can be used in different ways in agent-based simulation; ${ }^{6,7}$ it is possible to divide these contributions into two main tasks: i) Ex ante, where machine learning and data mining techniques can be used to achieve the one kind of intelligent behaviour that combines the data of past executions of simulation learning from experience and tuning some initial parameters of the simulation to a local maximum; ii) Ex post, where final results of a simulation are analysed using data mining techniques to uncover interesting patterns in data, helping to better model the behaviour of the overall systems. Note that the behaviour of the system is usually more than the sum of the parts and is not described in the first phase of the simulation task. Data mining could be used to create a model that is supported by statistical evidence that could be an initial hypothesis about the system.

\section{Conclusions}

This paper has a theoretical aim; its purpose is about introducing a hybrid simulation technique to be applied to the pharmacoeconomic analysis of ozone therapy. In particular, the approach of pharmacoeconomics is that of comparing the outcome of a traditional therapy with that of a different therapy. Ozone therapy is very interesting for medicine, since the effects and actions of $\mathrm{O}_{3}$ are mainly positive and beneficial for many different diseases. This makes ozone therapy as a very good candidate to be studied with the approach of pharmacoeconomics. While costs (both direct, indirect and intangible) are very important for deciding which therapy should be favoured, also other factors have to be taken into account like, for instance, lives saved, life years after therapy, quality of life and so on. This is why traditional approaches to pharmacoeconomics could come short (e.g. statistics); that's where simulation could be an effective method for evaluating a broader range of outcomes. For this reason, two simulation paradigms are discussed in this work, namely discrete event process simulation - to simulate those parts of the process which are well known and easily mouldable as a flow of actions and if/then clauses - and agent based simulation - to represent those parts or interactions where only general rules are known and the emergent aggregate behaviour is not determined a priori but rather coming out from the whole system and its ever-changing essence. So the combination of these two approaches can be a powerful analytical method to be applied to pharmacoeconomics in general and to ozone therapy in particular, as a case study. Moreover, in order to select the data to be used in such simulations, but also to analyse results coming from them, a data mining approach is proposed.

\section{References}

1. Stahl JE. Modelling methods for pharmacoeconomics and health technology assessment: an overview and guide. Pharmacoeconomics 2008;26:131.

2. Valdenassi L, Franzini M, Richelmi P, Bertè F. Ossigenoozono terapia. Atti del I Convegno Simfer, 2005 Nov 22, Pavia, Italy.

3. Ostrom TM. Computer simulation: the third symbol system. J Experim Soc Psychol 1988;24:381-92.

4. Remondino M. Agent based process simulation and metaphors based approach for enterprise and social modelling. Proceedings of the 4th International Conference on Agent Based Simulation. Ghent, Belgium: SCS European Publishing House; 2003. pp 93-7.

5. Remondino M. Agent based process simulation for management and economics. Econ Complex 2003. Available from: www.cs.unibo.it/cianca/wwwpages/labspo/Remondino.pdf

6. Remondino M, Correndo G. Data mining applied to agent based simulation. In: Proceedings of the 19th European Conference on Modelling and Simulation. Riga, Latvia; 2005. pp 1-4.

7. Remondino M, Correndo G. Mabs validation through repeated execution and data mining analisys. Intern J Simulation: Syst Sci Technol 2006;7:6. 